\title{
Correction
}

\section{Correction: Zhao et al., "Muscle Yap Is a Regulator of Neuromuscular Junction Formation and Regeneration"}

In the article "Muscle Yap Is a Regulator of Neuromuscular Junction Formation and Regeneration" by Kai Zhao, Chengyong Shen, Yisheng Lu, Zhihui Huang, Lei Li, Christopher D. Rand, Jinxiu Pan, Xiang-Dong Sun, Zhibing Tan, Hongsheng Wang, Guanglin Xing, Yu Cao, Guoqing Hu, Jiliang Zhou, Wen-Cheng Xiong, and Lin Mei, which appeared on pages 3465-3477 of the March 29, 2017 issue, the authors regrettably noticed a typo in proof in the $y$-axis label of Figure $3 D$ which reads "custer" instead of "cluster". This correction does not affect the conclusions of the paper. Figure 3 has been corrected on the online PDF version and displayed below.

A

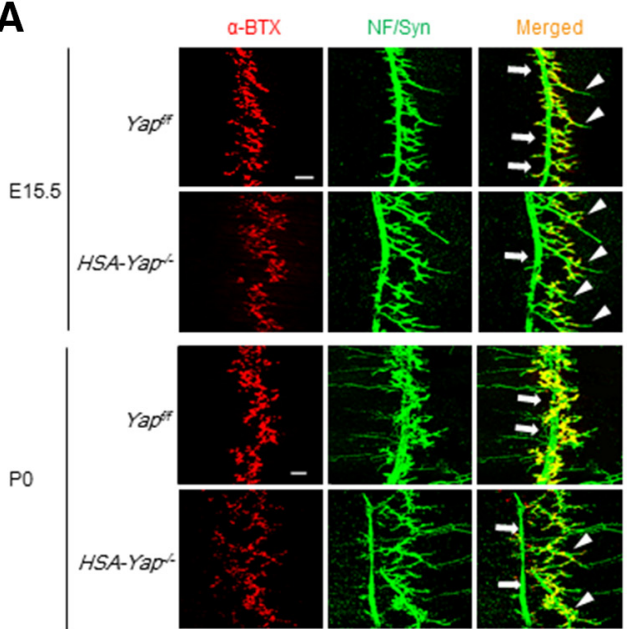

G

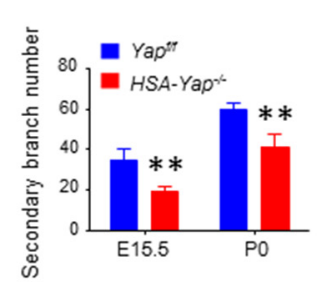

$\mathbf{H}$
B

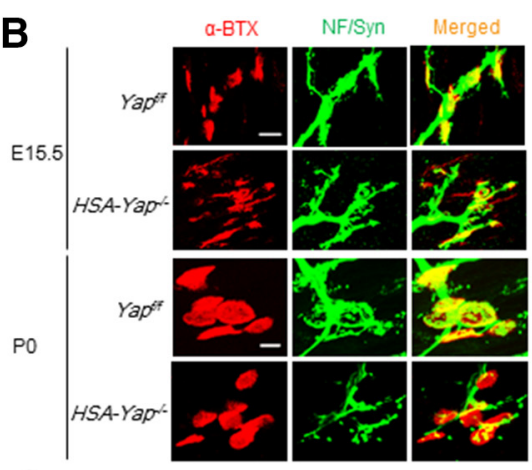

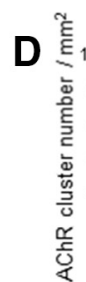

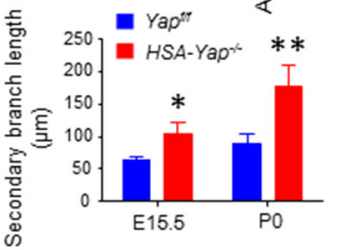

C

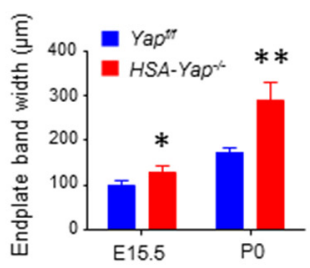

$\mathbf{F}$

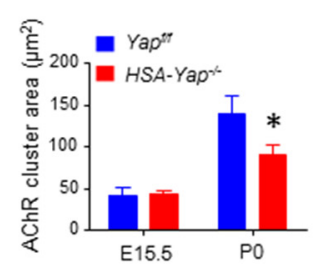

